
\title{
28 Research Suare \\ Relationship of femoral offset and clinical outcomes of femoral neck fracture patients following bipolar hip arthroplasty
}

\section{Sung Soo Kim}

Dong-A University Medical Center

Hyeon Jun Kim ( $\nabla$ hyeonjun@dau.ac.kr)

Dong-A University Medical Center https://orcid.org/0000-0002-3775-6421

\section{Seung Yup Lee}

Dong-A University Medical Center

\section{Research article}

Keywords: Hip joint, Femoral neck fractures, Hemiarthroplasty, Femoral offset

Posted Date: March 24th, 2020

DOI: https://doi.org/10.21203/rs.3.rs-18701/v1

License: (c) (i) This work is licensed under a Creative Commons Attribution 4.0 International License.

Read Full License 


\section{Abstract}

\section{Purpose}

This study aimed to investigate the relationship between femoral offset(FO) and clinical outcomes of patients with femoral neck fracture following bipolar hip arthroplasty(BHA).

Materials and Methods

This study recruited 520 patients who underwent BHA for femoral neck fracture from December 2003 to September 2018 with a minimum follow-up of one year. Excluding those with unclear medical records, a previous surgical history on the affected or contralateral hip, a congenital deformity and preoperative trauma history of the hip, high-energy multiple trauma, a history of neurodegenerative disease or cerebrovascular disease, a total of 77 patients were included in the analysis. The subjects were 54 females and 23 males. The mean age of patients was 74.6(65-95) years old and the mean follow-up period was 30.5 (12-136) months. For clinical assessment, postoperative pain visual analogue scale(VAS) and Harris hip score (HHS) were analyzed at each follow-up period. For radiological assessment, the difference in leg length discrepancy (LLD) and FO was measured. FO nearest to the real value was calculated using the values corrected for magnification and rotation errors on the anteroposterior view of plain radiographs taken at the first year of follow-up. In statistical analyses, the statistical significance between FO and clinical outcomes was analyzed using the Pearson correlation tests with SPSS v. 25.0. A value of $p<0.05$ was considered statistically significant.

Results

The difference in mean FO postoperatively was $6.7( \pm 4.8) \mathrm{mm}$, and the difference in mean postoperative LLD was 4.9 ( \pm 3.4$)$. The mean HHS was 77.1 $( \pm 7.7), 82.3( \pm 8.6), 83.4( \pm 7.7)$, and 86.4( \pm 6.7$)$ at $1 \mathrm{st}, 3 \mathrm{rd}, 6 \mathrm{th}$ and 12th postoperative months, respectively. In the analysis using the Pearson correlation coefficient, the correlation coefficient between FO and HHS in 1 -year follow-up was -0.38 , and a significant outcome was found $(p=0.001)$. Of HHS domain, the correlation coefficient for function in 1-year follow-up was -0.42 , revealing a significant outcome $(\mathrm{p}=0.0001)$.

Conclusion

There was a stastically significant correlation between clinical oucomes and FO difference at 1 year after BHA in patients with femoral neck fractures older than 65 years.

\section{Introduction}

The incidence of hip fracture is steadily increasing with a rising life expectancy ${ }^{1)}$. Intertrochanteric fracture and femoral neck fracture account a large percentage of all hip fractures in older patients, and hip joint arthroplasty is commonly performed. Undisplaced femoral neck fractures that are not severe are typically treated with internal fixation, but displaced fractures at the time of admission to the hospital are 
more common. Hip arthroplasty is immediately performed to patients at high risk for fixation failure due

to old age ${ }^{2)}$. In recent years, total hip arthroplasty (THA) is increasingly performed in patients with femoral neck fracture, and favorable clinical outcomes have been reported ${ }^{3-4}$. However, since THA is more extensive surgery and has the higher risk of dislocation compared to bipolar hip arthroplasty(BHA), BHA remains the preferred treatment in Korea ${ }^{5}$. In hip arthroplasty, biomechanical restoration is important and femoral offset (FO) is the most critical factor towards a successful hip replacement ${ }^{6-11)}$. A large number of studies have explored FO in THA, but only a few studies have focused on BHA. Therefore, we aimed to investigate the correlation between FO and clinical outcomes in patients who underwent BHA for femoral neck fracture with a minimum follow-up of one year.

\section{Materials And Methods}

\section{Subjects}

This study recruited 520 patients aged 65 years and over and who underwent BHA after being diagnosed with femoral neck fracture from December 2003 to September 2018. The subjects were chosen based on the following exclusion criteria. (Fig. 1)

- Those with a follow-up of less than 1 year

- Those with unclear medical records and difficulty communicating

- Those with a previous surgical history on the contralateral hip

- Those with a previous surgical history on the affected hip

- Those with a congenital deformity and preoperative trauma history of the hip

- Those with high-energy multiple trauma at the time of femoral neck fracture

- Those with a history of neurodegenerative disease or cerebrovascular disease

A total of 77 patients were included in the analysis. The subjects were 54 females and 23 males. The mean age of patients was $74.6(65-95)$ years old and the mean follow-up period was $30.5( \pm 23.8)$ months. (Table 1).

2. Surgical methods and rehabilitation methods

All operations were performed in the lateral position using a posterolateral approach. Preoperative templating was performed for predicting the size of implant used in surgery. Before implant insertion during surgery, intraoperative portable X-ray was taken to confirm the size and location of the implant. The shuck test and stability test were done to assess stability including dislocation and impingement, and the actual implant was placed. A cementless femoral stem was used in all operations. Considering patient's systemic condition, surgeons allowed quadriceps exercise started from the second postoperative day. Standing and walker ambulation were begun in patients who actively flexed the hip. Walker ambulation was maintained and then shifted to cane ambulation in patients at high risk for slip down. In 
outpatient follow-up visits, full weight bearing was permitted in patients with good muscle strength and at low risk for slip down.

\section{Clinical results}

To identify the impact of the change in FO on clinical results of patients with femoral neck fracture after BHA, pain VAS at $1^{\text {st }}$ postoperative year and HHS at $1^{\text {st }}, 3^{\text {rd }}, 6^{\text {th }}$ and $12^{\text {th }}$ months postoperatively were assessed. HHS was categorized into pain domain with a maximum of 44 points and functional domain with a maximum of 47 points.

\section{Radiological assessment}

The difference of FO was defined as the change in the FO of the contralateral normal hip on preoperative 3-dimensional computed tomography (3D CT) compared to that of the operated side on plain radiographs obtained 1 year after BHA. To enhance the accuracy of FO of the normal side, 3D CT (Aquilion $\mathrm{ONE}^{\circledR}$, Canon Medical Systems, Otawara, Japan) was used and the perpendicular distance from the center of rotation of the femoral head to the femoral shaft axis was measured in the 3D axial, sagittal and coronal planes using the PACS system (Marosis M-view ${ }^{\mathrm{TM}}$ 5.4, Marotech, Seoul, Korea). (Fig. 2)

FO of the operated hip was measured in the anterior-posterior pelvic views obtained 1 year after the surgery using the PACS system. The projected femoral offset (FOP) was defined as the perpendicular distance from the hip center of rotation to the femoral shaft axis on plain X-ray images. Since measurement errors occur on radiographs due to magnification rates by projection distance from the $\mathrm{X}$ ray source to the anatomical structure, magnification correction factor (MCF) was calculated and used to reduce errors in the real FO value ${ }^{12)}$. Since the shape of the acetabular cup is a hemisphere, the real cup diameter (CDR) can be obtained via correction for error rates according to projection distance because the maximum diameter of the cup represents the true hemispherical diameter on a plane in orthographic projection due to the geometrical nature. The real cup diameter (CDR) is the projected cup diameter (CDP) measured on plain radiographs using the PACS multiplied by MCF. Therefore, MCF can be calculated using the following equation. (Fig. 3)

$\mathrm{MCF}=\mathrm{CDR} / \mathrm{CDP}$

Using this formula, the real leg length discrepancy (LLDR) was calculated by multiplying MCF by the projected leg length discrepancy (LLDP), the measurement of the perpendicular distance between a horizontal line drawn through the inferior aspect of the teardrops and the prominent point of the lesser trochanter on plain radiographs taken 1 year after the surgery.

$\operatorname{LLDR}=\operatorname{LLDP} \times \mathrm{MCF}$

A caution is to be careful when assessing $\mathrm{FO}$ on plain radiographs because the more internally rotated the hip is, the smaller the real offset will appear ${ }^{13-16)}$. To minimize measurement errors resulted from the 
degree of rotation, rotation correction factor (RCF) according to hip rotation was obtained using the method verified by Lechler et. al. ${ }^{16)}$. The following equation was used by assessing the tangent function of the targeted implant yangle $(\mathrm{yl})$ and the projected yangle $(\mathrm{\gamma P})$ of the femoral stem on plain radiographs. (Fig. 4)

$\mathrm{RCF}=\tan (\mathrm{Yl}) / \tan (\mathrm{YP})$

Finally, the following equation was used to calculate the rotation-corrected FO (FOR) nearest to the true value.

$\mathrm{FOR}=\mathrm{FOP} \times \mathrm{RCF} \times \mathrm{MCF}$

\section{Statistical Analysis}

All statistical analyses were performed using SPSS v. 25.0 (IBM Co.,Armonk, NY, USA). The Pearson correlation tests were done to identify the statistical significance in postoperative changes in FO, LLD, VAS, HHS pain, and HHS function at follow-ups. The absolute value of the Pearson correlation coefficient higher than 0.3 was interpreted as having a clear linear relationship. A value of $p<0.05$ was considered statistically significant.

\section{Results}

The mean preoperative FO on the contralateral normal side was $42.5( \pm 9.1) \mathrm{mm}$ in patients with femoral neck fracture. The mean FO was $38.1( \pm 6.1) \mathrm{mm}, 1$ year after BHA. The mean difference in FO was 6.7 $( \pm 4.8) \mathrm{mm}$. The mean MCF was $0.88( \pm 0.04)$, and the mean RCF was $1.19( \pm 0.24)$. The mean LLD was $4.9( \pm 3.4) \mathrm{mm}$. (Table 1) The mean HHS was $77.1( \pm 7.7)$ in 1 month after BHA, and improved to 86.4 $( \pm 6.7)$ in 1 year after surgery. The mean score improved from $36.3( \pm 5.3)$ in 1 month to $40.8( \pm 3.1)$ in 1 year after surgery in the pain domain of the HHS. The mean score improved from $33.2( \pm 4.7)$ in 1 month to $38.6( \pm 3.9)$ in 1 year after surgery in the functional domain of the HHS. In the Pearson correlation tests, the Pearson correlation coefficient between FO and HHS in 1-year follow-up was -0.38 , showing a clear negative linear relationship and this result indicated a significant correlation $(p=0.001)$. (Fig. 5$)$

The correlation coefficient for HHS function domain in 1-1year follow-up was - 0.42 , revealing a higher correlation ( $p=0.0001)$. However, the correlation coefficient for HHS pain domain in 1-year follow-up was -0.21 , showing no significant correlation $(p=0.06)$. Moreover, no significance was found in all correlations between LLD and clinical outcomes during the follow-up period (Table 2)

\section{Discussion}

The number of elderly patients with hip fracture is continuously increasing, and hip arthroplasty is commonly performed in older patients with femoral head fracture ${ }^{1,2)}$. BHA was first introduced by Bateman and Giliberity in 1974. Unlike unipolar hemiarthroplasty, BHA is designed to provide the inner 
bearing between the femoral head and the polyethylene surface and the outer bearing between the acetabular cup and the acetabulum ${ }^{2-4}$ ). Compared with THA, BHA is more frequently performed type of arthroplasty because this procedure is a less complicated surgery without acetabular replacement and has the advantage of an increased jumping distance that lowers the risk for dislocation ${ }^{5}$. Like in THA, the FO is important in BHA. FO is the perpendicular distance between the center of rotation of the femoral head and a line drawn down the centre of the femoral shaft, and this is a critical in biomechanical studies on artificial hip joints ${ }^{6-11}$ ). The hip joint is the lever arm of body weight and the abductor mechanism and hip abductor muscles act as the lever arm extending from the lateral aspect of the greater trochanter and the femoral head, and a greater FO reduces forces required by the abductors by increasing the abductor moment arm. Multiple studies have explored the relationship between FO and clinical outcomes in THA. Bjordal et al. ${ }^{9)}$ compared clinical results between two groups with increased FO by more than $5 \mathrm{~mm}$ and decreased FO by more than $5 \mathrm{~mm}$ at 1 year follow-up after THA, and reported significantly poor functional results including pain severity, HHS and others in the group with a reduction of FO by more than $5 \mathrm{~mm}$. De Fine et al. ${ }^{10}$ ) investigated the association of FO with bearing surface wear, implant loosening and dislocation rate in a systematic review on FO restoration during THA. Two of three articles revealed a statistical association between bearing surface wear and FO restoration and showed no statistical significance between implant loosening and dislocation rate.

Sariali et al. ${ }^{11)}$ found out that 6- to 12-mm decrease in FO after THA altered the gait in $15 \%$ of all articles on the effect of FO modification after THA. In particular, a lower maximal swing speed and range of motion while walking were significantly excellent in the group with restored FO.

A large number of previous studies have shown the clinical outcomes of FO in THA. On the contrary, only a few studies have explored FO in BHA. The reasons are as follows. First, long-term follow-up is difficult in patients with femoral neck fractures because most patients are old and mortality rates range between $28-30 \%$ within the first year of follow-up ${ }^{1}$. Second, it is not easy to detect significance difference in clinical results because older patients frequently have walking difficulty from before the surgery and gait disturbance due to comorbidities. In addition, there are challenges in an accurate assessment of FO.

Several studies have reported that errors can occur due to internal rotation and projection distance in measurement of hip offsets after THA, and an underestimation of the offset value on plain radiographs due to internal rotation has been commonly accepted ${ }^{12-16)}$.

In this study, magnification error by projection distance was adjusted with MCF. Bae et al. ${ }^{12)}$ suggested that magnification rate was smaller as projection distance shortened, and magnification rate of plain Xray of the femoral head was approximately $11.4 \%$. In the present study, MCF was $0.88( \pm 0.04)$ and we verified that the size needed to be reduced by about $12 \%$ on plain X-ray to be nearest to the actual size.

Merle et al. ${ }^{13)}$ suggested that FO was frequently underestimated by approximately $13 \%$ on plain radiographs due to internal rotation of the hip, and recommended careful templating using $\mathrm{CT}$ because measurement errors in preoperative templating in THA may influence surgical outcomes. Weber et al. ${ }^{14)}$ 
proposed that the measurement of FO with CT was more accurate than with plain radiographs, by revealing a mean error of $1.0( \pm 2.0)$.

Pasquier et al. ${ }^{15)}$ asserted and proved in their study that CT was a more accurate way for measuring FO and LLD. Therefore FO on the preoperative contralateral normal side was assessed using 3D CT in our study. This was possible because most patients with femoral neck fracture are admitted to hospital via emergency room and radiographic images are obtained by performing routine CT scanning before admission to emergency room. However, FO is assessed using plain radiography at postoperative followups. The reasons for insufficient radiographic data are avoidance of high radiation doses from CT scans, high cost, patient compliance and others. To calculate postoperative FO nearest to the real value in plain radiographs, RCF was used. Philipp et al. ${ }^{16)}$ calculated RCF using centrum column diaphyseal angle(CCD) of inserted implants including the femoral stem and lag screw in 222 patients with proximal femoral nailing. In the Friedman test, there was no significant difference in the mean value between the mean FO measured using CT and the mean FO obtained using RCF on plain radiographs.

In our study, the exclusion criteria were reinforced to find out definite differences in clinical outcomes during the follow-up period. Although this may reflect selection bias, it was appropriate for our study in which we aimed to identify even a small difference in clinical results based on patients' clear responses to postoperative inquiries. For example, there would be insignificant difference in clinical scores even 1 year after surgery in patients with inability to walk due to hemiparesis caused by a preoperative history of cerebral infarction. In addition, the evaluation of clinical symptoms and improvement would be less accurate in patients having difficulty communicating due to dementia.

Rösler et al. ${ }^{17)}$ compared clinical outcomes such as Barthel index, Tinetti score and others in 250 patients with proximal femur fracture in the 1-year follow-up by classifying patients into three groups based on the MMSE. As a result, significantly unfavorable clinical outcomes were shown in the group with severe dementia with a score of less than 11 on the MMSE and a history of cerebrovascular accident.

There are several clinical assessment parameters in exploring the difference in clinical results of patients after hip arthroplasty including HHS, hip disability and osteoarthritis outcome score (HOOS), Oxford hip score (OHS), Lequesne index of severity for osteoarthritis of the hip (LISOH), timed up and go test (TUG), IAPL, Barthel index, Tinetti score and others ${ }^{18}$ ). In the present study, HHS and VAS were used in the assessment of clinical outcomes, because HHS is the most commonly used tool to measure hip function in domestic and international literature and the evaluation criteria are easier for patients to understand. In a study on 50 patients who received hip arthroplasty and surgeons, Mahomed et al. ${ }^{19)}$ reported that items recorded by patients were almost the same as items recorded by surgeons in the HHS survey questionnaire.

The VAS has been used extensively in many studies. Data collection rate was good because VAS was one of the requirements in EMR in our institution. The mean VAS score was $1.5( \pm 1.1)$ in the first follow-up year, revealing no significant difference with the mean HHS pain score of $40.8( \pm 3.1)$. 
In the current study, the Pearson correlation coefficient between FO and HHS within 1-year follow-up was -0.38 , and a significant negative correlation was observed $(p=0.001)$. The Pearson coefficient for HHS function domain in 1-year follow-up was -0.42 , revealing a higher correlation $(p=0.0001)$. However, the Pearson coefficient for HHS pain domain in 1 -year follow-up was -0.21 , showing no significant correlation $(p=0.06)$. Through these results, we have inferred that FO after the first postoperative year was not correlated with pain severity, but was correlated with HHS function in femoral neck fracture patients with BHA. No significant difference in HHS functional domain at $1^{\text {st }}, 3^{\text {rd }}$, and $6^{\text {th }}$ postoperative months seems to be resulted from the fact that functional domain was measured when functional rehabilitation was not fully completed and functional outcome is believed to be affected by limitations in daily activities such as pain.

$\mathrm{Ji}$ et al. ${ }^{20)}$ measured rotation-free FO using preoperative CT scans of the contralateral hip joint, the FO was changed by less than $20 \%$ in $77 \%$ of patients and more than $20 \%$ in $23 \%$ of patients, indicating clinically unfavorable outcomes.

Buecking et al. ${ }^{21)}$ investigated clinical results by subdividing FO into rotation-corrected FO, relative FO, and contralateral FO with 1-year follow-up in 126 patients who underwent BHA due to femoral neck fractures. In Spearman correlation analysis, there was a significant association of FO with HHS and instrumental activities of daily living(IADL).

Lakstein et al. ${ }^{22)}$ addressed that there were difficulties in verifying the association between the restoration of LLD and FO and functional outcome at 1-year follow-up, and rehabilitation in old age was largely affected by patient's general medical and mental status. On the contrary, Hartel et al. ${ }^{23)}$ reported that anatomical restoration of an artificial hip joint had an insignificant effect on short-term functional outcome by showing in-hospital mortality of $5.7 \%$ and one-year mortality of $30 \%$ among elderly patients who received BHA for femoral neck fractures. Differences with our study is, the average age of the patient group was 84(78-94) years, relatively higher than in our study. And the exclusion criteria were only those with preoperative proximal femur fracture and a surgical history on the contralateral side. For this reason, it is believed patients having no difference between pre- and post-operative clinical results appear to account for a large percentage. Furthermore, since FO was not corrected for rotation, this likely that the measured FO was underestimated.

The strength of this study is that FO was obtained nearest to the real value by correcting for rotation and magnification errors in FO measurements. Moreover, this study strictly specified preoperative exclusion criteria to clarify clinical outcomes in compliance with the study purpose and to exclude obscure cases in determining any difference between pre- and post-operative results. We also aimed to identify the changes in pre-and post-operative results by investigating clinical outcomes according to the follow-up period. However, there are some limitations to note. First, this study has relatively small sample size. Second, the study was not conducted by a single surgeon and retrospectively.

\section{Conclusion}


There was a stastically significant correlation between clinical oucomes and FO difference at 1 year after $\mathrm{BHA}$ in patients with femoral neck fractures older than 65 years. Therefore, along with increasing life expectancy in aging societies, more careful attention is needed in FO restoration in femoral neck fracture patients.

\section{Declarations}

\section{Abbreviations}

FO: Femoral offset

BHA: Bipolar hip arthroplasty

VAS: Visual analogue scale

HHS: Harris hip score

LLD: Leg length discrepancy

THA: Total hip arthroplasty

PACS:Picture archiving and communication system

3D CT: 3-dimensional computed tomography

FOP: The projected femoral offset

MCF: Magnification correction factor

CDR: Real cup diameter

CDP: Projected cup diameter

LLDR: Real leg length discrepancy

LLDP: Projected leg length discrepancy

CCD: Centrum column diaphyseal angle

RCF: Rotation correction factor

FOR: Rotation corrected FO

\section{Ethics approval and consent to participate}

Not applicable. 


\section{Consent for publication}

Not applicable.

\section{Availability of data and materials}

All data generated or analyzed during this study are included in this published article.

\section{Competing interests}

The authors declare that they have no competing interests.

\section{Funding}

This study was supported by the Dong-A University research fund.

\section{Authors' contributions}

HJK designed the study and analyzed the data. SYL performed statistical analysis and language editing. KSS was a major contributor in writing the manuscript. All authors read and approved the final manuscript.

\section{Acknowledgements}

Not applicable.

\section{References}

1. Kanis JA, Odén A, McCloskey EV, Johansson H, Wahl DA, Cooper C. A systematic review of hip fracture incidence and probability of fracture worldwide. Osteoporos Int. 2012;23:2239-56

2. Bhandari M, Devereaux PJ, Tornetta P 3rd, Swiontkowski MF, Berry DJ, Haidukewych G, et al. Operative management of displaced femoral neck fractures in elderly patients. An international survey. J Bone Joint Surg Am. 2005;87:2122-30.

3. Burgers PT, Van Geene AR, Van den Bekerom MP, Van Lieshout EM, Blom B, Aleem IS, et al. Total hip arthroplasty versus hemiarthroplasty for displaced femoral neck fractures in the healthy elderly: a meta-analysis and systematic review of randomized trials. Int Orthop. 2012;36:1549-60.

4. Ullmark G. Femoral head fractures: hemiarthroplasty or total hip arthroplasty? Hip Int. 2014;24 Suppl:e2-4. 
5. Yoo JI, Cha YH, Kim JT, Park CH. Clinical Outcomes of Bipolar Hemiarthroplasty versus Total Hip Arthroplasty: Assessing the Potential Impact of Cement Use and Pre-Injury Activity Levels in Elderly Patients with Femoral Neck Fractures. Hip pelvis. 2019;31:63-74

6. Lecerf G, Fessy MH, Philippot R, Massin P, Giraud F, Flecher X, et al. Femoral offset: anatomical concept, definition, assessment, implications for preoperative templating and hip arthroplasty. Orthop Traumatol Surg Res. 2009;95:210-9.

7. Flecher $X$, Ollivier M, Argenson JN. Lower limb length and offset in total hip arthroplasty. Orthop Traumatol Surg Res. 2016;102 Suppl:S9-20.

8. Mahmood SS, Mukka SS, Crnalic S, Wretenberg P, Sayed-Noor AS. Association between changes in global femoral offset after total hip arthroplasty and function, quality of life, and abductor muscle strength. A prospective cohort study of 222 patients. Acta Orthopaedica. 2016; 87:36-41

9. Bjordal F, Bjorgul K. The role of femoral offset and abductor lever arm in total hip arthroplasty. J Orthop Traumatol 2015;16:325-30.

10. De Fine M, Romagnoli M, Toscano A, Bondi A, Nanni M, Zaffagnini S. Is there a role for femoral offset restoration during total hip arthroplasty? A systematic review. Orthopaedics \& Traumatology: Surgery \& Reserch. 2017;103:349-55.

11. Sariali E, Klouche S, Mouttet A, Pascal-Moussellard H. The effect of femoral offset modification on gait after total hip arthroplasty. Acta Orthop. 2014;85:123-7.

12. Bae YJ, Kang KS, Tae SK, Jung YB, Lee HJ, Jin WJ. Roentgenographic study on shape and size of the femoral head of normal korean adults. Journal of korean orthopedics. 1995;30:607-11

13. Merle C, Waldstein W, Pegg E, Streit MR, Gotterbarm T, Aldinger PR, et al. Femoral offset is underestimated on anteroposterior radiographs of the pelvis but accurately assessed on anteroposterior radiographs of the hip. J Bone Joint Surg Br. 2012;94:477-82.

14. Weber M, Woerner ML, Springorum HR, Hapfelmeier A, Grifka J, Renkawitz TF. Plain Radiographs Fail to Reflect Femoral Offset in Total Hip Arthroplasty. J Arthroplasty. 2014;29:1661-5.

15. Pasquier G, Ducharne G, Ali ES, Giraud F, Mouttet A, Durante E. Total hip arthroplasty offset measurement: is C T scan the most accurate option? Orthopaedics \& Traumatology. 2010;96:367-75

16. Lechler P, Frink M, Gulati A, Murray D, Renkawitz T, Bucking B, et al. The influence of hip rotation on femoral offset in plain radiographs. Acta Orthop. 2014;85:389-95.

17. Rösler A, Krause T, Niehuus $C$, von Renteln-Kruse W. Dementia as a cofactor for geriatric rehabilitation-outcome in patients with osteosynthesis of the proximal femur: a retrospective, matched-pair analysis of 250 patients. Archives of Gerontology and Geriatrics. 2009;49:e36-39

18. Nilsdotter A, Bremander A. Measures of Hip Function and Symptoms. Arthritis Care \& Research. 2011;63 Suppl:S200-7

19. Mahomed NN, Arndt DC, McGrory BJ, Harris WH. The Harris Hip Score: Comparison of Patient SelfReport With Surgeon Assessment. J Arthroplasty. 2001;16:575-80. 
20. Ji HM, Won SH, Han J, Won YY. Does femoral offset recover and affect the functional outcome of patients with displaced femoral neck fracture following hemiarthroplasty?. Injury Int J Care Injured. 2017;48:1170-4

21. Buecking B, Boese CK, Bergmeister VA, Frink M, Ruchholtz S, Lechler P. Functional implications of femoral offset following hemiarthroplasty for displaced femoral neck fracture. Int Orthop. 2016;40:1515-21

22. Lakstein D, Atoun E, Wissotzky O, Tan Z. Does restoration of leg length and femoral offset play a role in functional outcome one year after hip hemiarthroplasty?, Injury Int J Care injured. 2017;48:158993

23. Hartel M, Arndt M, Eulenburg CZ, Petersen JP, Rueger JM, Hoffmann M. Restoration of hip architecture with bipolar hemiarthroplasty in the elderly: does it affect early functional outcome? Arch Orthop Trauma Surg. 2014;134:31-8

\section{Tables}

Table.1. dermographic characteristics and radiologic measurement in femoral neck fracture patients following bipolar hip arthroplasty

\begin{tabular}{ll}
\hline Variables & \\
\hline Age at the fractures in years $74.6(65-95)$ \\
Gender & $23(29.9 \%)$ \\
\hline Male & $54(70.1 \%)$ \\
\hline Female & $30.5(12-136)$ \\
\hline Follow up period in months & \\
\hline & $42.5( \pm 9.1)$ \\
\hline Preoperative FO *(mm) & $38.1( \pm 6.1)$ \\
\hline Postoperative FO(mm) & $6.7( \pm 4.8)$ \\
\hline FO difference(mm) & $0.88( \pm 0.04)$ \\
\hline MCF $^{\dagger}$ & $1.19( \pm 0.24)$ \\
\hline RCF $^{\ddagger}$ & $4.9( \pm 3.4)$ \\
\hline LLDR $^{\S}(\mathrm{mm})$ &
\end{tabular}

Values are presented as number only, mean \pm standard deviation (range), or number (\%). 
$\mathrm{FO}^{*}$ : femoral offset, $\mathrm{MCF}^{\dagger}$ : magnification correction factor

$\mathrm{RCF}^{\ddagger}$ : rotation correction factor, $\mathrm{LLDR}^{\S}$ : leg length discrepancy

Table.2. Clinical results according to follow up period and correlation between femoral offset difference and leg length discrepancy

\begin{tabular}{|c|c|c|c|c|}
\hline & & \multicolumn{3}{|c|}{$\mathrm{FO}^{*}$ difference $\mathrm{LLD}^{\dagger}$} \\
\hline & & $\mathrm{PCC}^{\ddagger}$ & $P$-value & PCC $P$-value \\
\hline 1month HHS & $\S 77.1( \pm 7.7)$ & -0.17 & 0.12 & 0.180 .11 \\
\hline Pain & $36.3( \pm 5.3)$ & -0.05 & 0.64 & 0.160 .15 \\
\hline Function & $33.2( \pm 4.7)$ & -0.22 & 0.05 & 0.160 .15 \\
\hline 3month HHS & $82.3( \pm 8.6)$ & -0.18 & 0.11 & 0.220 .07 \\
\hline Pain & $38.5( \pm 4.6)$ & -0.10 & 0.35 & 0.230 .06 \\
\hline Function & $35.5( \pm 5.9)$ & -0.19 & 0.08 & 0.170 .12 \\
\hline 6month HHS & $83.4( \pm 7.7)$ & -0.15 & 0.19 & 0.220 .06 \\
\hline Pain & $39.3( \pm 3.6)$ & 0.03 & 0.79 & 0.210 .07 \\
\hline Function & $36.1( \pm 4.7)$ & -0.25 & 0.06 & 0.210 .08 \\
\hline 12month HHS & $\mathbf{S} 86.4( \pm 6.7)$ & -0.38 & 0.001 & 0.050 .64 \\
\hline Pain & $40.8( \pm 3.1)$ & -0.21 & 0.06 & -0.080 .47 \\
\hline Function & $38.6( \pm 3.9)$ & -0.42 & 0.0001 & 0.130 .26 \\
\hline Last VAS" & $1.5( \pm 1.1)$ & 0.26 & 0.12 & -0.100 .35 \\
\hline
\end{tabular}

$\mathrm{FO}^{*}$ : femoral offset, $\mathrm{LLD}^{\dagger}$ : leg length discrepancy, $\mathrm{PCC}^{\ddagger}$ : Pearson correlation coefficient

HHS $^{\S}$ : Harris hip score, VAS" ${ }^{\|}$visual analogue score 
Figures

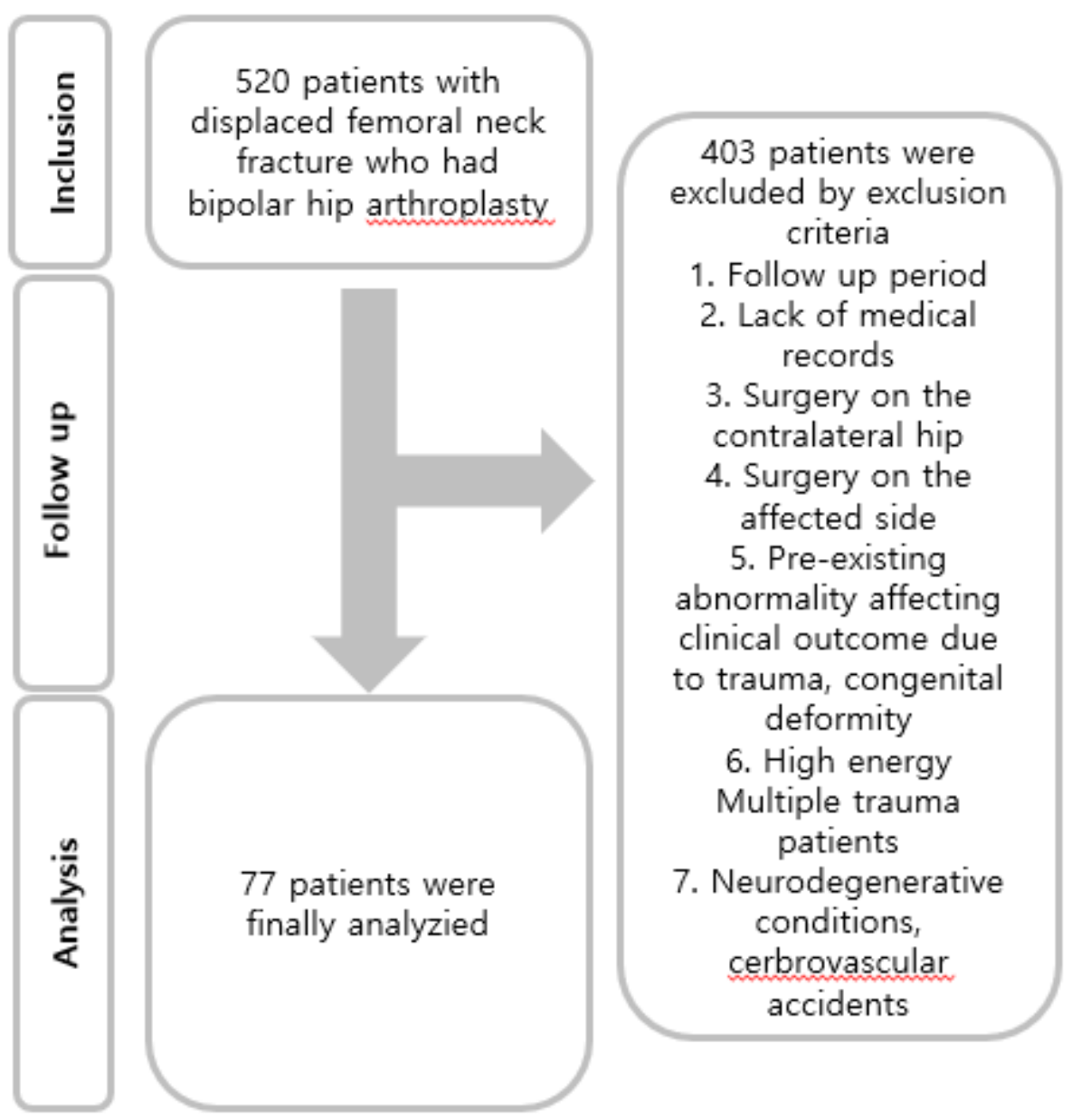

Fig. 1. Flow diagram patient follow up and exclusion criteria

\section{Figure 1}

Flow diagram patient follow up and exclusion criteria 


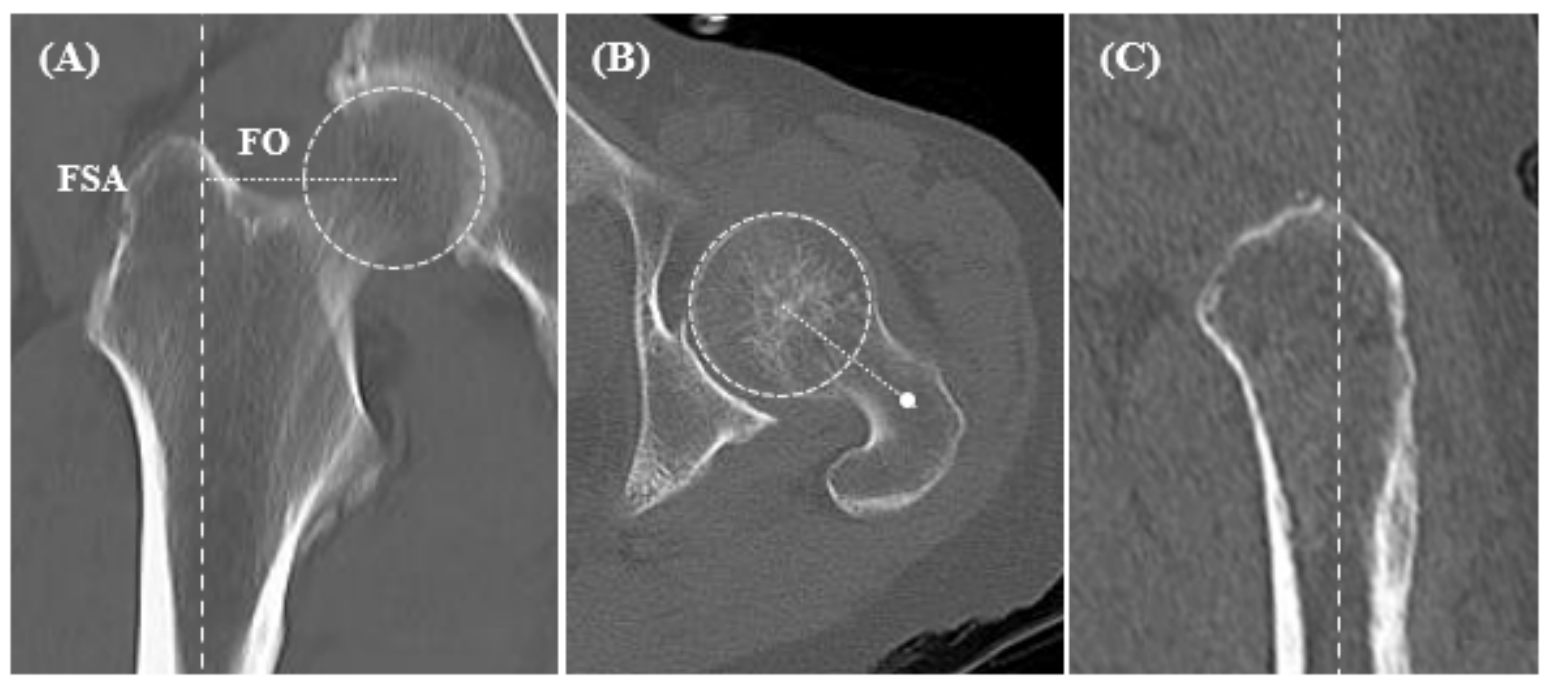

Fig. 2. Measurement of femoral offset with 3D CT. The femur shaft axis(dashed line) and center of femoral head are determined by determined by (A)coronal, (B)axial, (C)saggital plane. Femoral offset is determined by the perpendicular line between the femur shaft axis and the center of the femoral head.

FSA femur shaft axis, FO femoral offset

\section{Figure 2}

Measurement of femoral offset with 3D CT. The femur shaft axis(dashed line) and center of femoral head are determined by determined by $(A)$ coronal, $(B)$ axial, (C)saggital plane. Femoral offset is determined by the perpendicular line between the femur shaft axis and the center of the femoral head. FSA femur shaft axis, FO femoral offset 


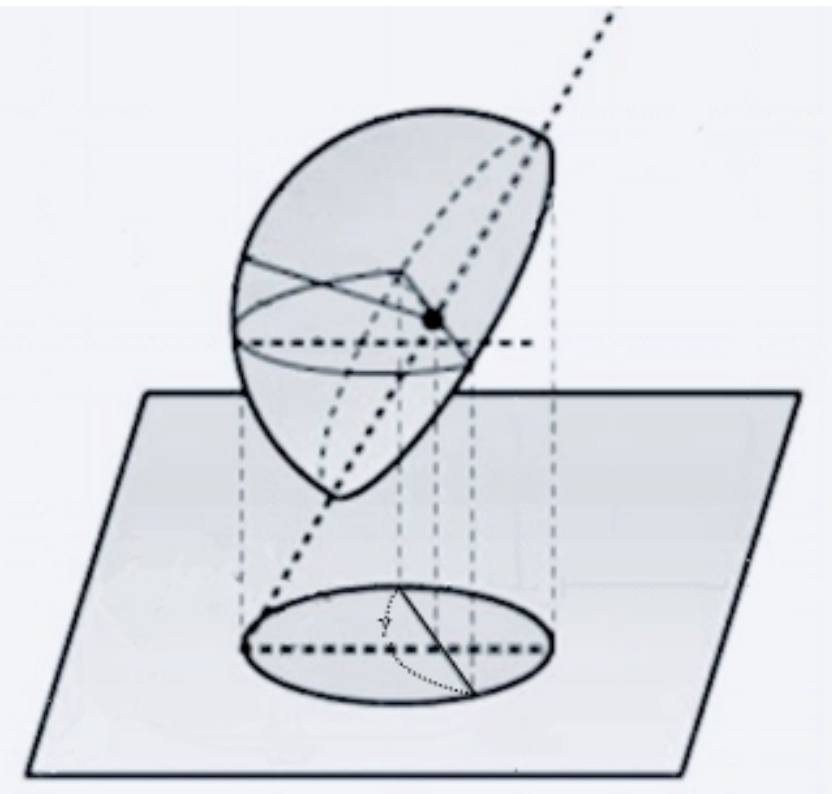

Fig. 3. Diagram orthogonal projection of hemisphere. By the hemisphere's geometrical nature, the maximum diameter $(\gamma)$ of the orthogonal projection of the hemisphere is the actual diameter of the hemisphere.

\section{Figure 3}

Diagram orthogonal projection of hemisphere. By the hemisphere's geometrical nature, the maximum diameter(Y) of the orthogonal projection of the hemisphere is the actual diameter of the hemisphere. 
Fig.4. Measurement of projection and rotation corrected femoral offset and leg length discrepancy.

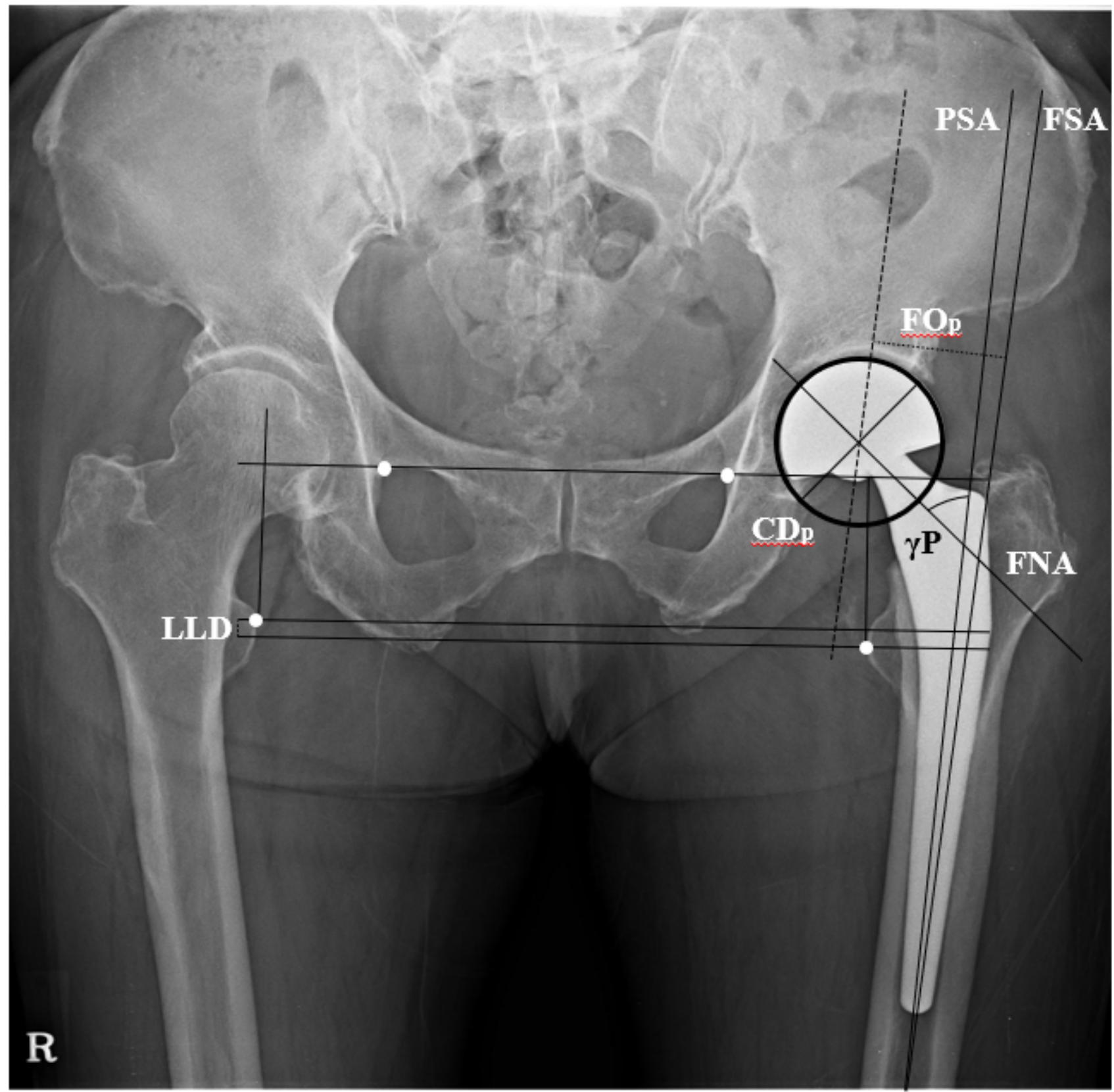

Femoral offset is measured as the horizontal distance between the hip center of rotation and the femoral shaft axis. LLD is measured as the vertical distance between a horizontal line connecting teardrops and the most prominent point of the lesser trochanter.

LLD leg length discrepancy, CDP projected cup diameter, FNA femoral neck axis, FOP projected femoral offset, FSA femoral shaft axis, PSA prosthesis shaft axis, $\gamma \mathrm{P}$ projected gamma angle of the implanted femoral component

\section{Figure 4}

Measurement of projection and rotation corrected femoral offset and leg length discrepancy. Femoral offset is measured as the horizontal distance between the hip center of rotation and the femoral shaft axis. LLD is measured as the vertical distance between a horizontal line connecting teardrops and the most prominent point of the lesser trochanter. LLD leg length discrepancy, CDP projected cup diameter, 
FNA femoral neck axis, FOP projected femoral offset, FSA femoral shaft axis, PSA prosthesis shaft axis, YP projected gamma angle of the implanted femoral component

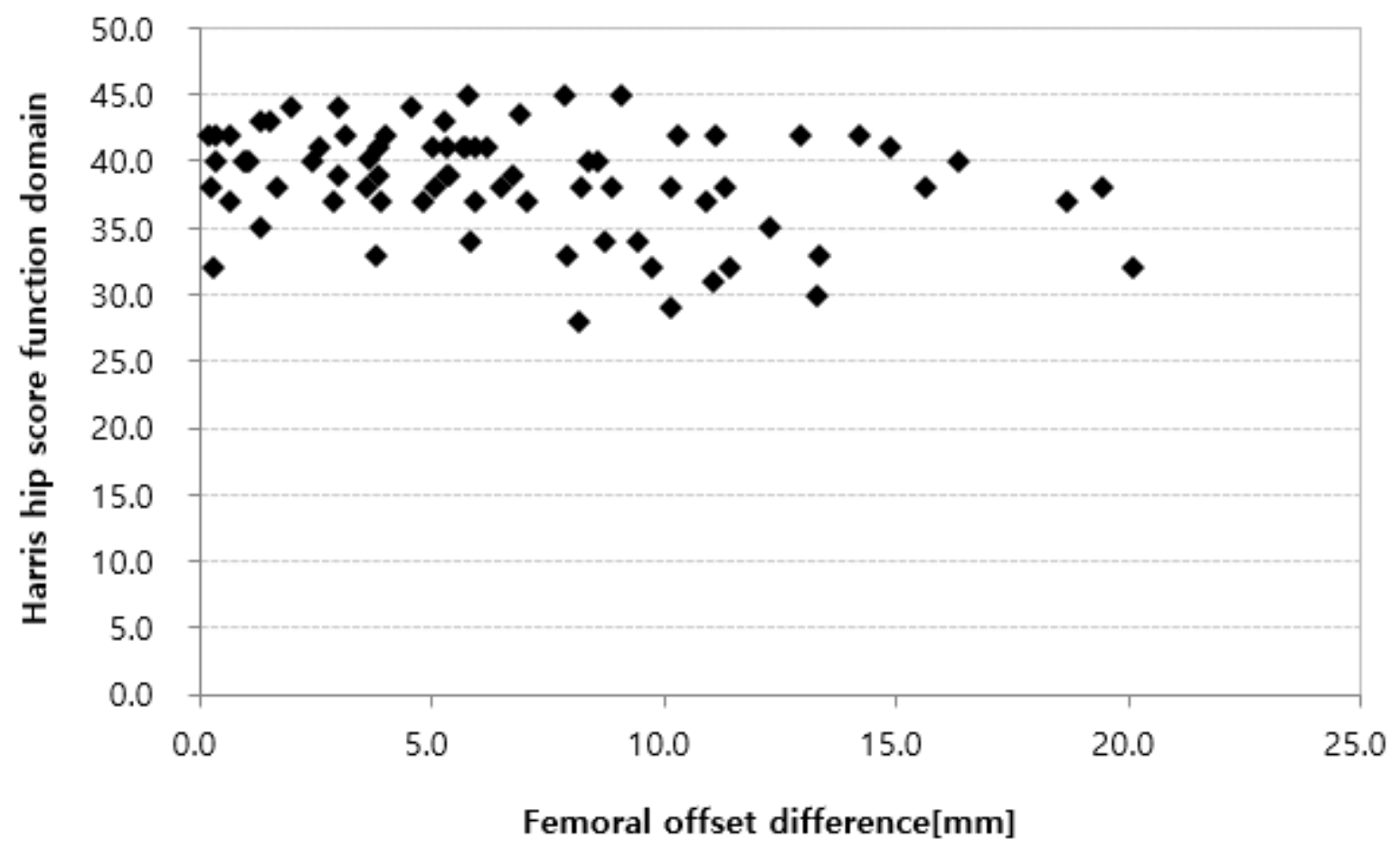

Fig. 5. The scatter graph between the Harris hip score function domain and femoral offset difference in femoral neck fracture patients following bipolar hip arthroplasty

\section{Figure 5}

The scatter graph between the Harris hip score function domain and femoral offset difference in femoral neck fracture patients following bipolar hip arthroplasty 